\title{
045 DISEASE PROFILING IN ASIAN FILIPINOS
}

Maria Luisa de Guzman Daroy. Research and Biotechnology Group, St. Luke's Medical Center, Quezon City, Philippines

10.1136/bmjopen-2015-forum2015abstracts. 45

Background The integration of medical informatics, biobanking, and genetic profiling of human subjects is a key paradigm in disease-focused research. This approach has evolved through the 
years in response to the dearth of comprehensive information on complex diseases, the need for standards and quality checks for the collection of clinical samples, and the scarcity of genetic data on Filipinos.

Objectives This study program aims to utilize disease-focused informatics, biobanking and genomics to obtain molecular profiles associated with important non-communicable diseases in Asian Filipino patients.

Methods Serum or plasma fractions are collected and DNA extracted from the buffy coat. These derivatives are then aliquoted and stored in a biofreezer of the biobank. At the different steps of processing and archiving, specimen quality is assessed periodically to ensure its usefulness for research. Meticulous documentation is performed, and inventory records are electronically archived to facilitate specimen retrieval from the biobank. Samples and information records are electronically barcoded at two levels to ensure the patient confidentiality and if required, either de-linking or anonymization of the source, while keeping intact data links to the specimens and the subsequent research results Specimens are withdrawn from the biobank and processed to extract, purify and analyze cellular, nucleic acid, protein, or metabolite components.

Result Patient DNA were examined for genetic variations, specifically single nucleotide polymorphisms (SNPs), indels, nucleotide repeats, and mutations in various genes that have been previously reported to be associated with a disease. A pharmacogenetics study of SNPs in several genes related to drug use is also underway. These polymorphisms are identified using Sanger DNA sequencing, PCR-RFLP, Taqman Real-time PCR and High Resolution Melt assays. The genomic data obtained is then stored in genomics databases which are linked to the clinical information through a second barcode. Allele and genotype frequencies are calculated and usefulness of a genetic variant as a risk factor, diagnostic biomarker or target molecule for novel drugs is assessed using statistical and computational tools.

Conclusion These profiles of genetic variations in Filipino patients thus represent the genome of present-day Asian Filipinos. The integrated approach provides in-depth data coverage for the manifestations of coronary artery disease, hypertension, Alzheimer's dementia or a specific cancer in the human subject, as well as the dynamics of the biological background in which the disease is expressed. This complementary system of data banking and biobanking is an indispensable strategy for grounding genomics research to its translational application and clinical outcomes. 